\title{
Double-Vitrectomy for Optic Disc Pit Maculopathy
}

\author{
Francesco Pichi $^{a} \quad$ Mariachiara Morara $^{b}$ Chiara Veronese $^{b}$ \\ Andrea Lembo $^{a}$ Lucia Vitale ${ }^{a}$ Antonio P. Ciardella ${ }^{b}$ \\ Paolo Nucci ${ }^{\mathrm{a}}$ \\ ${ }^{a}$ San Giuseppe Hospital, University Eye Clinic, Milan, and ${ }^{b}$ Ophthalmology Unit, \\ Policlinico Sant'Orsola-Malpighi, Bologna, Italy
}

\section{Key Words}

Optic pit maculopathy $\cdot$ Internal limiting membrane peeling $\cdot$ Gas tamponade

\begin{abstract}
Background: The origin of the fluid and precise pathophysiology of optic pit maculopathy remain unclear. It has been suggested that submacular fluid originates either from vitreous or cerebrospinal fluid. We report a case of optic pit maculopathy which was unsuccessfully treated with vitrectomy and internal limiting membrane (ILM) peeling, and subsequently resolved with revision of vitrectomy and gas tamponade.
\end{abstract}

Methods: We report a case of optic disc pit maculopathy, well documented by spectraldomain optical coherence tomography, before and after pars plana vitrectomy with ILM peeling, and its revision with gas tamponade.

Results: After ILM peeling, there was no improvement either in visual acuity or in the tomographic aspect of the retina. A revision of the surgery was then needed and gas tamponade was performed, which resulted in a complete resolution of the optic pit maculopathy.

Conclusion: The absence of improvement after ILM peeling during the first surgical procedure, accompanied by resolution of the clinical picture with gas tamponade during the second surgical procedure, sustained the hypothesis of a subarachnoidal origin of the fluid.

\section{Introduction}

A pit of the optic disc was first described by Whiethe [1] in 1882 as 2 ellipsoid blackish cavities in the optic nerve in a 62-year-old woman. He differentiated the cavities from pigmentation by careful observation of the course of the vessel at the disc [2-4]. Although the patient described by Whiethe had a glaucoma in the other eye, for 
many years, the primary importance of optic disc pits in glaucoma assessment [5] was a differential diagnosis of normal-tension glaucoma [6-8].

Several authors described incident pits of the optic disc in open-angle glaucoma (OAG), both clinically and histologically, demonstrating an association with glaucoma progression, presence in low-tension glaucoma, and limitation of the pit to within the substance of the lamina cribrosa [9-12]. Subsequently, a number of authors confirmed these findings in clinic-based case-control studies [13-15].

The pathogenesis of the macular abnormalities as well as the source of the fluid continue to be controversial, with various theories proposed for both the source of the abnormal fluid as well as the conduit of the fluid [2,15-22].

We report a case of optic pit maculopathy in OAG unsuccessfully treated with vitrectomy and internal limiting membrane (ILM) peeling that subsequently resolved with revision of vitrectomy and gas tamponade.

\section{Methods}

An 89-year-old woman, followed in our Glaucoma Clinic for primary OAG in both eyes, was referred to our Retina Clinic for painless, progressive reduction of central vision and macular edema in her right eye. Her left eye had no light perception because of uncontrolled glaucoma. When first examined, her visual acuity was 20/200 in the right eye. An anomalous optic disc with an optic pit, focal peripapillary pigmentary changes, and diffuse macular swelling consistent with edema or retinoschisis were present (ig. 1).

Optical coherence tomography (OCT) images obtained with the Spectralis HRA + OCT (version 1.5.12.0; Heidelberg Engineering, Heidelberg, Germany) showed fluid in the outer nuclear layer and retinal detachment which did not connect to the optic disc (fig. 1a). The OCT images could not detect any definite communication between the schisis-like separation and vitreous cavity or subarachnoid space, but confirmed the presence of an optic pit (fig. 1b). Initially, fluid from the pit caused an elevation of the nerve fiber layer leading to a schisis-like inner retinal separation. Subsequently, an outer layer detachment occurred, possibly due to the movement of fluid towards the outer segment.

Neither posterior vitreous detachment nor vitreomacular traction was observed. Her bestcorrected visual acuity (BCVA) continued to decline during the following month; therefore, after the informed consent of the patient, small-gauge (25-gauge) pars plana vitrectomy was performed with elevation of the posterior hyaloid, which was attached at the optic nerve and throughout the posterior pole. The ILM was removed after posterior vitreous detachment induction. Neither gas tamponade nor laser photocoagulation was performed during the primary procedure, nor was an attempt made to drain the fluid. Not performing gas tamponade in this case allowed elimination of need for prone positioning after surgery as well as of any complications related to fluid-air exchange or gas tamponade. In addition, the early postoperative events were studied better with OCT in the fluid-filled eye than in the gas-filled eye.

Spectral-domain (SD)-OCT examination at 1 week and 1 month postoperatively (fig. 2) did show a slight decrease of retinal thickness (fig. $2 b$ ) consistent with a slight reabsorption of the cystic fluid in the inner retinal layers nasally to the macula; however, the retinal detachment persisted and, additionally, an outer layer macular hole developed beneath the inner layer (fig. 2a). Accordingly, the patient's BCVA remained stable at 20/200.

The patient then underwent revision of vitrectomy, fluid-air exchange with aspiration, and placement of gas tamponade. Postoperative SD-OCT scanning demonstrated that the sharp contour of the retinal elevation adjacent to the optic disc and inner retinoschisis-like separation were reduced immediately (fig. $3 \mathrm{a}$ ) and BCVA improved to 20/25. These findings remained stable at 3 months after the second surgery (fig. $3 b$ ). 


\section{Discussion}

Pits of the optic disc have been described in case reports and case series since the late 19th century [1]. In 2007, Healey and Mitchell [23] conducted a population-based survey of 3,654 individuals and found an overall pit prevalence of $0.19 \%$ and a prevalence of OAG of $2.8 \%$. In both congenital and acquired optic pits, the source of the fluid has been open to discussion.

Brown et al. [19] conducted experiments on Collie dogs, injecting India ink into the vitreous cavity and observing it in the subretinal space. However, direct communication between the liquefied vitreous and the subretinal space has not been demonstrated conclusively using OCT.

It is improbable that the fluid originates from leaky blood vessels at the base of the pit because there does not appear to be a breakdown of the blood-retina barrier, as demonstrated by the lack of leakage during fluorescein angiography.

The fluid could be cerebral spinal fluid (CSF). In 1996, an OCT-based study by Krivoy et al. [21] demonstrated that there was direct communication between the schisis cavity and the subarachnoid space. On the one hand, this is strong supporting evidence that CSF can move towards the submacular space via the subarachnoid space. On the other hand, CSF might not be the source because of the difference in hydrostatic pressure between CSF and intraocular pressure [2].

Theoretically, an ideal procedure for reattaching the macular schisis associated with an optic disc pit would be one that reroutes the flow of the fluid from the pit. Conservative management used to be the initial recommendation as $25 \%$ of the optic pit maculopathies resolve spontaneously. However, the poor visual outcome of the natural course of these spontaneously resolved detachments [24-26] has made a more aggressive surgical approach the preferred treatment modality for most retinal specialists. Previous attempts at repair have included laser photocoagulation near the nerve. However, laser energy is principally absorbed by the pigment in the retina pigment epithelium and choroid, creating a deep scar, while the most pronounced changes in optic disc maculopathy do not occur deep in the retina adjacent to the nerve. The surgical goal in this strategy appears to be the creation of an adhesion to limit the passage of the fluid from the region of the pit into the retina; however, this procedure has a high rate of recurrence and morbidity. In recent years, the release of traction (either vitreous or ILM induced) has been considered a very important factor in the management of patients with optic disc pit maculopathy and was also the first choice in our case, with pars plana vitrectomy associated with ILM peeling. Unfortunately, in our case, there was no anatomical or functional success. Spaide et al. [27] recently proposed a new technique suggesting surgery of the inner retinal fenestration as a possible treatment.

The buoyancy of an air bubble may compress the retina ab interno. We postulate that with the second surgical intervention, there might have been a posterior shift in the fluid to the subarachnoid space of the optic nerve rather than drainage from the vitreous cavity, which, if present, should have happened after ILM removal during the first vitrectomy. After gas tamponade, rather than moving to the vitreous, the fluid might have shifted away from the peripapillary region, either anteriorly into the more 
peripheral retina or posteriorly into the retrolaminar subarachnoidal space of the optic nerve. The former is less likely because of the supine position of the patient and the specific gravity of fluid versus air. A decrease in the macular volume would require the anterior shift to be significant enough to shift the fluid outside the analyzed area. Thus, it appears that the retinal fluid associated with the optic disc pit was redirected into the retrolaminar subarachnoid space under pressure of the gas bubble, which further elevated intraocular pressure above intracranial pressure.

\section{Conclusion}

The observations in this case report support a functional connection between the subarachnoid space and the intraretinal schisis. Indeed, revision of vitrectomy with gas tamponade resolved the macular detachment and shifted the subretinal fluid. This suggested that the fluid may had come into the intraretinal layers along the vessels from the optic disc, which is porous because of the disorganized tissue, and the lamina cribrosa, also defective within the pit because of OAG. Although this connection between the subretinal and the subarachnoid spaces appears most likely given our findings, additional mechanisms for the observations should be considered.

The optimal treatment of optic disc pit maculopathy is unclear. Numerous approaches have been suggested, including laser photocoagulation and pars plana vitrectomy with various adjuncts (e.g. laser, gas exchange, ILM peeling, and inner retinal fenestration [27]). Our patient demonstrated a progressive decrease in schisis volume with an improvement in visual acuity following vitrectomy and gas tamponade.

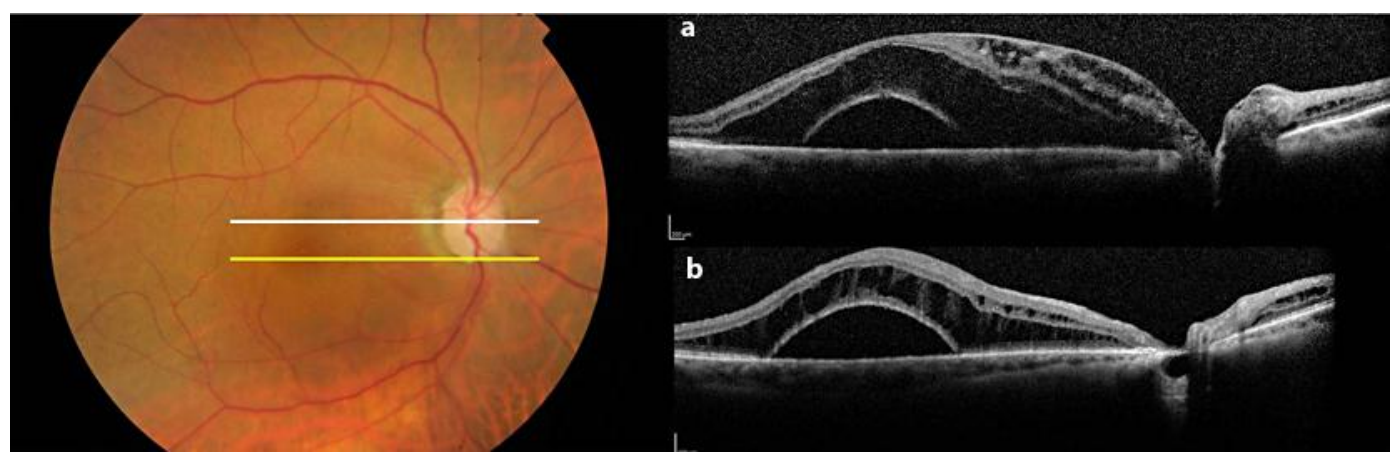

Fig. 1. A fundus color photo shows a localized white/yellow oval depression in the inferotemporal segment of the optic disc and diffuse macular swelling. An SD-OCT scan through the fovea (a) shows cystic accumulation of fluid in the inner retinal layers adjacent to the disc, a schisis-like accumulation of fluid in the outer nuclear layer, and an underlying retinal detachment. This scan cannot detect any definite communication between the retinal separation and the optic nerve, but an SD-OCT scan taken at the inferior margin of the disc (b) shows a colobomatous pit of the nerve head. 

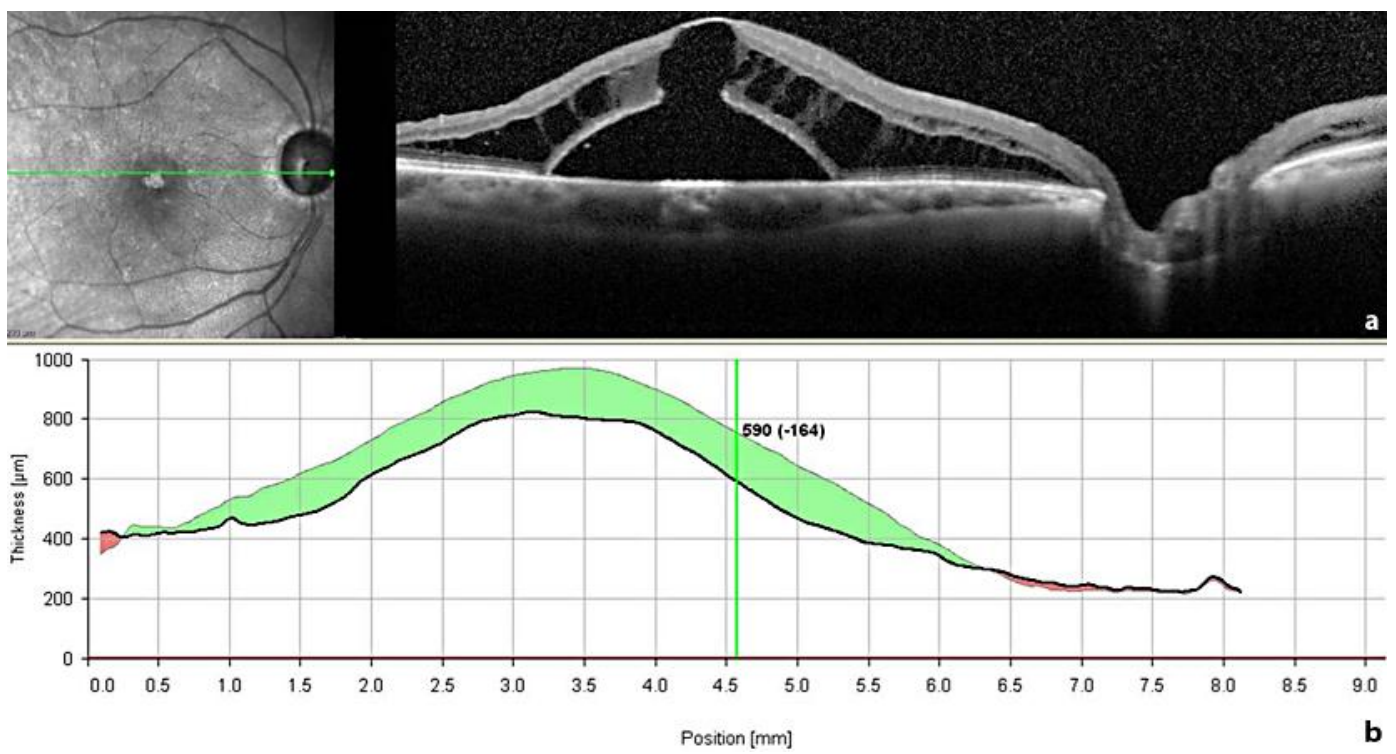

Fig. 2. An SD-OCT scan through the fovea taken 4 weeks after the first surgical procedure (pars plana vitrectomy and ILM peeling) (a) shows a slight reduction of the inner retinal cysts near the disc, whereas the outer layer schisis-like separation and the retinal detachment did not improve.

Moreover, an outer layer macular hole developed beneath the schisis. The decrease in central retinal thickness of $164 \mu \mathrm{m}$ (b) is rather due to a displacement of the fluid than to reabsorption of it.

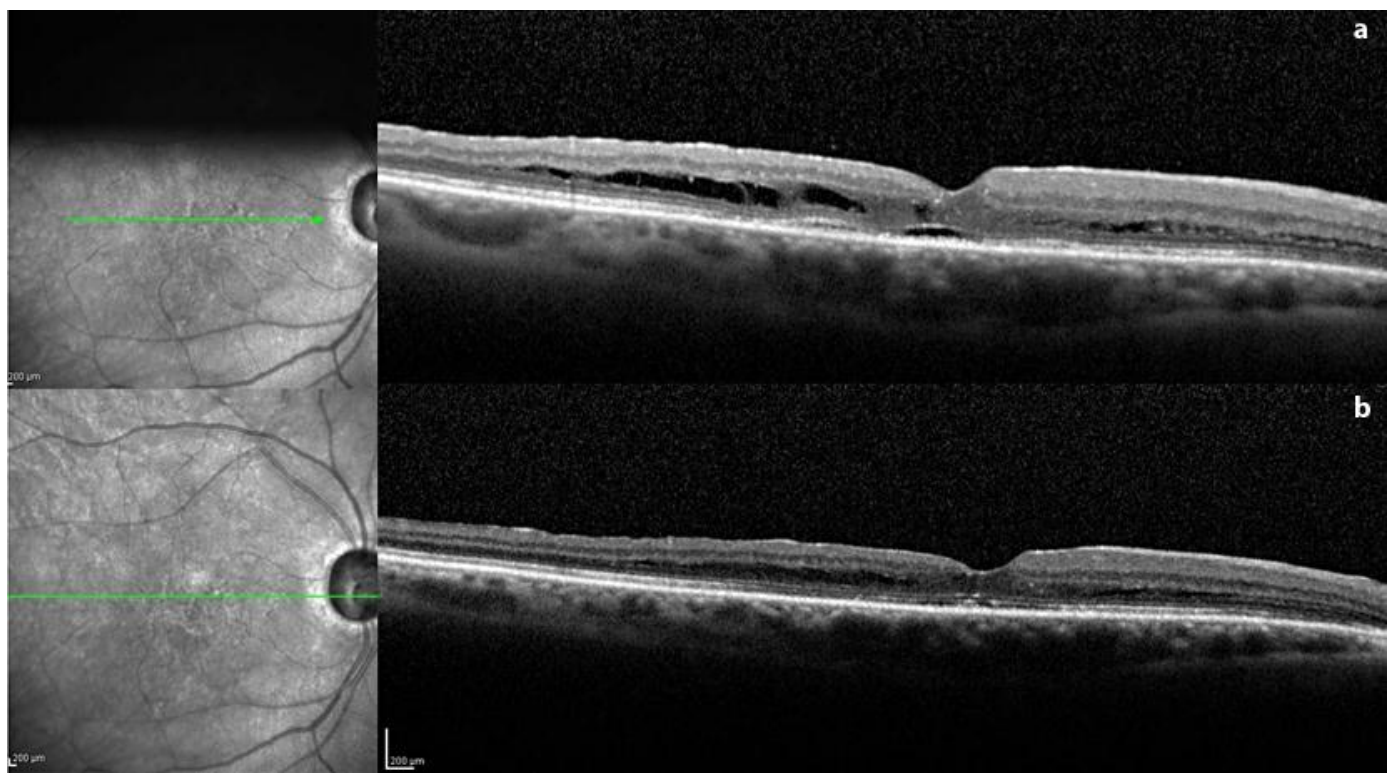

Fig. 3. After revision of vitrectomy with gas tamponade, an SD-OCT scan of the same point made 1 week postoperatively (a) shows complete resolution of the retinal detachment and of the schisislike separation of the outer layer, with a restitutio ad integrum of the foveal depression and of the external hyperreflective bands. A minimal amount of cystic fluid persists in the parafoveal outer layer, which could no longer be detected at 1 month (b). 


\section{References}

1 Wiethe T: Ein Fall von angeborener Difformität der Sehnervenpapille. Arch f Augenh 1882;11:14-19.

$>2$ Gass JD: Serous detachment of the macula. Secondary to congenital pit of the optic nervehead. Am J Ophthalmol 1969;67:821-841.

-3 Lincoff H, Lopez R, Kreissig I, Yannuzzi L, Cox M, Burton T: Retinoschisis associated with optic nerve pits Arch Ophthalmol 1988;106:61-67.

4 Moon SJ, Kim JE, Spaide RF: Optic pit maculopathy without inner retinal schisis cavity. Retina 2006;26:113-116

5 Gayer MO: Acquired hole in the disc. Br J Ophthalmol 1951;35:437-439.

6 Sandvig K: Pseudoglaucoma of autosomal, dominant inheritance. A report on three families. Acta Ophthalmol (Copenh) 1961;39:33-43.

-7 Lichter PR, Henderson JW: Optic nerve infarction. Trans Am Ophthalmol Soc 1977;75:103-121.

-8 Simpson DE: Optic nerve pit. J Am Optom Assoc 1987;58:118-120.

-9 Greear JN: Pits, or crater-like holes, in the optic disc. Arch Ophthalmol 1942;28:467-483.

10 Smith JL: The optic nerve workup. Trans Sect Ophthalmol Am Acad Ophthalmol Otolaryngol 1977;83:778-785

11 Duke-Elder S: Congenital Deformities. System of Ophthalmology. London, Kimpton, 1964, pp 679-682.

12 Radius RL, Maumenee AE, Green WR: Pit-like changes of the optic nerve head in open-angle glaucoma. Br J Ophthalmol 1978;62:389-393.

-13 Javitt JC, Spaeth GL, Katz LJ, et al: Acquired pits of the optic nerve. Increased prevalence in patients with low-tension glaucoma. Ophthalmology 1990;97:1038-1043.

14 Nduaguba C, Ugurlu S, Caprioli J: Acquired pits of the optic nerve in glaucoma: prevalence and associated visual field loss. Acta Ophthalmol Scand 1998;76:273-277.

15 Ugurlu S, Weitzman M, Nduaguba C, et al: Acquired pit of the optic nerve: a risk factor for progression of glaucoma. Am J Ophthalmol 1998;125:457-464.

16 Spaeth GL: A new classification of glaucoma including focal glaucoma. Surv Ophthalmol 1994;38(suppl):S9-S17.

17 Cashwell LF, Ford JG: Central visual field changes associated with acquired pits of the optic nerve. Ophthalmology 1995;102:1270-1278.

18 Oh JY, Park KH: Analysis of optic disc change using the Heidelberg retina tomograph in an acquired pit of the optic nerve. Ophthalmic Surg Lasers Imaging 2004;35:325-327.

$\checkmark 19$ Brown GC, Shields JA, Patty BE, Goldberg RE: Congenital pits of the optic nerve head. I. Experimental studies in collie dogs. Arch Ophthalmol 1979;97:1341-1344.

20 Postel EA, Pulido JS, McNamara JA, Johnson MW: The etiology and treatment of macular detachment associated with optic nerve pits and related anomalies. Trans Am Ophthalmol Soc 1998;96:73-88.

-21 Krivoy D, Gentile R, Liebmann JM, Stegman Z, Rosen R, Walsh JB, Ritch R: Imaging congenital optic disc pits and associated maculopathy using optical coherence tomography. Arch Ophthalmol 1996;114:165170.

-22 Kuhn F, Kover F, Szabo I, Mester V: Intracranial migration of silicone oil from an eye with optic pit. Graefes Arch Clin Exp Ophthalmol 2006;244:1360-1362.

-23 Healey PR, Mitchell P: The prevalence of optic disc pits and their relationship to glaucoma. J Glaucoma 2008;17:11-14.

24 Sugar HS: An explanation for the acquired macular pathology associated with congenital pits of the optic disc. Am J Ophthalmol 1964;57:833-835.

-25 Sugar HS: Congenital pits of the optic disc and their equivalents (congenital colobomas and colobomalike excavations) associated with submacular fluid. Am J Ophthalmol 1967;63:298-307.

26 Yuen CH, Kaye SB: Spontaneous resolution of serous maculopathy associated with optic disc pit in a child: a case report. J AAPOS 2006;6:330-331.

27 Spaide RF, Fisher Y, Ober M, Stoller G: Surgical hypothesis: inner retinal fenestration as a treatment for optic disc pit maculopathy. Retina 2006;26:89-91. 